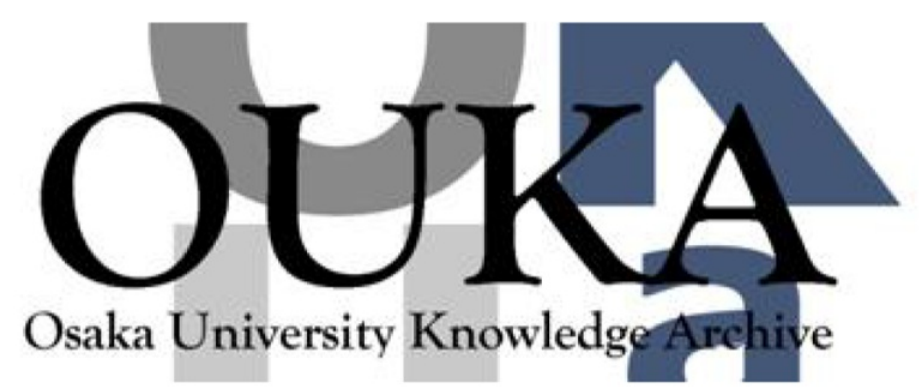

\begin{tabular}{|c|l|}
\hline Title & A variational problem for Affine Connections \\
\hline Author(s) & Kobayashi, 0samu \\
\hline Citation & Archiv der Mathematik. 86(5) p. 464-p. 469 \\
\hline Issue Date & $2006-05$ \\
\hline oaire:version & VoR \\
\hline URL & https://hdl. handle.net/11094/26340 \\
\hline rights & \\
\hline Note & \\
\hline
\end{tabular}

Osaka University Knowledge Archive : OUKA

https://ir. Library. osaka-u. ac. jp/

Osaka University 


\title{
A Variational Problem for Affine Connections
}

\author{
Osamu Kobayashi
}

Abstract. We give a variational problem for affine connections which characterizes the Riemannian connection of an Einstein metric of negative scalar curvature.

\section{Introduction}

Let $\mathcal{A}(M)$ denote the sapce of all torsion free affine connections of a compact connected manifold $M$, each of which preserves a volume element of $M$. Every affine connections is projectively equivalent to such a connection. For $\nabla \in \mathcal{A}(M)$ we can define the Ricci curvature tensor Ric. Since $\nabla$ preserves a volume element, Ric is a symmetric 2-tensor. Moreover using the volume element we can define the determinant det Ric of the Ricci tensor. We put for $\nabla \in \mathcal{A}(M)$

$$
E(\nabla)=\int_{M} \operatorname{det} \operatorname{Ric} d \mu \int_{M} d \mu
$$

It is easy to see that the right hand side is independent of the choice of a volume element $d \mu$ which is preserved by $\nabla$. Moreover $E(\nabla)$ depends on $\nabla$ differentiably. This functional may be compared with the normalized Einstein-Hilbert functional of the total scalar curvature of a Riemannian metric ([1]). In this paper we will show the following:

Theorem. Let $M$ be a compact connected manifold. Suppose $\nabla \in \mathcal{A}(M)$ is a critical point of E, and its Ricci curvature is negative semidefinite and negative definite somewhere. Then $\nabla$ is the Riemannian connection of an Einstein metric of $M$.

Remark. A Ricci flat connection $\nabla \in \mathcal{A}(M)$ is a critical point of the functional $E$. It is known [2; p. 211] that there is a Ricci flat affine connection which is not a Riemannian connection.

I would like to thank the referee for useful comments on the paper.

\section{Projective equivalence of affine connections}

Given two affine connections $\nabla$ and $\tilde{\nabla}$ on $M$, we say that they are projectively equivalent if geodesics ignoring their parameters are the same for $\nabla$ and $\tilde{\nabla}$. That is to say, there is a function $\lambda: T M \rightarrow \mathbf{R}$ such that

$$
\tilde{\nabla}_{X} X=\nabla_{X} X+2 \lambda(X) X
$$

for $X \in T M$. Then it is immediate to see that $\lambda$ is linear if we put $\lambda(0)=0$, and thus $\lambda$ is a smooth 1 -form of $M$.

We will see that any affine connection of $M$ is projectively equivalent to some connection in $\mathcal{A}(M)$. Let $\nabla$ be an arbitrary affine connection of $M$. Then an affine connection $\nabla^{\prime}$ defined as $\nabla_{X}^{\prime} Y=\frac{1}{2}\left(\nabla_{X} Y+\right.$ $\left.\nabla_{Y} X+[X, Y]\right)$ is a torsion free connection which is projectively equivalent to $\nabla$. So we assume $\nabla$ is torsion free. Let $\tilde{\nabla}$ be another torsion free connection which is projectively equivalent to $\nabla$. Then from (2.1),

$$
\tilde{\nabla}_{X} Y=\nabla_{X} Y+\lambda(X) Y+\lambda(Y) X
$$

Mathematics Subject Classification(2000): 53C05 
for some 1-form $\lambda$. Take a volume element $d \mu$ and $u \in C^{\infty}(M)$, we then have

$$
\tilde{\nabla}_{X}\left(e^{(n+1) u} d \mu\right)=e^{(n+1) u} \nabla_{X} d \mu+(n+1) e^{(n+1) u}(d u-\lambda)(X) d \mu,
$$

where $n=\operatorname{dim} M$. Hence if we choose $u=0$ and $\lambda$ to be such that $(n+1) \lambda(X) d \mu=\nabla_{X} d \mu$, we have $\tilde{\nabla} d \mu=0$, i.e., $\tilde{\nabla} \in \mathcal{A}(M)$.

The formula (2.2) says more and we have the following.

Lemma 2.1. Suppose that $\nabla, \tilde{\nabla} \in \mathcal{A}(M)$ are projectively equivalent. Then there is a $u \in C^{\infty}(M)$ such that

$$
\tilde{\nabla}_{X} Y=\nabla_{X} Y+(X u) Y+(Y u) X .
$$

Moreover if $\nabla d \mu=0$, then $\tilde{\nabla}\left(e^{(n+1) u} d \mu\right)=0$.

Corollary 2.2. Suppose $\nabla, \tilde{\nabla} \in \mathcal{A}(M)$. Then we have

$$
\tilde{\nabla}_{X} Y=\nabla_{X} Y+(X u) Y+(Y u) X+S(X, Y)
$$

where $u \in C^{\infty}(M)$ and $S=\left(S_{j k}^{i}\right)$ is a $(1,2)$-tensor such that $S_{j k}^{i}=S_{k j}^{i}$ and $S_{j i}^{i}=0$. The tensor $S$ is uniquely determined by $\nabla$ and $\tilde{\nabla}$, and $u$ is determined up to a constant.

Proof. Let $T=\tilde{\nabla}-\nabla$ be the difference of the two connections. Put $S_{j k}^{i}=T_{j k}^{i}-\frac{1}{n+1}\left(T_{j l}^{l} \delta_{k}^{i}+T_{k l}^{l} \delta_{j}^{i}\right)$. Then $\nabla+S \in \mathcal{A}(M)$ is projectively equivalent to $\tilde{\nabla}$. $\square$

Corollary 2.3. Denote by $\mathcal{V}(M)$ the space of smooth volume elements of $M$. Then we have a smooth map $\varphi: \mathcal{A}(M) \rightarrow \mathcal{V}(M)$ such that $\nabla \varphi(\nabla)=0$.

Proof. Fix $\nabla \in \mathcal{A}(M)$ and $d \mu \in \mathcal{V}(M)$ such that $\nabla d \mu=0$. Fix a point $x \in M$. Then any $\tilde{\nabla} \in \mathcal{A}(M)$ is given as in (2.3), and we can determine a unique $u$ on condition that $u(x)=0$. Then put $\varphi(\tilde{\nabla})=e^{(n+1) u} d \mu$. [

3. The variational formula for the functional $E$

First we note that the Ricci curvature of $\nabla \in \mathcal{A}(M)$ is a symmetric 2-tensor.

Let $\omega \in \Lambda^{n} T_{x}^{*} M \backslash\{0\}$ be a volume form at $x \in M$. Define $\omega^{*} \in \Lambda^{n} T_{x} M$ by $\omega\left(\omega^{*}\right)=1$. Then we have $(a \omega)^{*}=\frac{1}{a} \omega^{*}$ for $a \in \mathbf{R} \backslash\{0\}$. Thinking of the Ricci tensor as Ric: $T_{x} M \rightarrow T_{x}^{*} M$, we define $\operatorname{det}_{\omega}$ Ric as

$$
\operatorname{Ric}^{*} \omega^{*}=\left(\operatorname{det}_{\omega} \operatorname{Ric}\right) \omega \text {. }
$$

For $a \in \mathbf{R} \backslash\{0\}$, we have $\operatorname{det}_{a \omega}$ Ric $=\frac{1}{a^{2}} \operatorname{det}_{\omega}$ Ric. In particular $\operatorname{det}_{-\omega} \operatorname{Ric}=\operatorname{det}_{\omega}$ Ric. Hence we can define $\operatorname{det}$ Ric $=\operatorname{det}_{d \mu}$ Ric with respect to a volume element $d \mu$.

Now suppose $\nabla d \mu=\nabla d \mu^{\prime}$. Then $d \mu^{\prime}=a d \mu$ for some positive constant $a$ since $M$ is connected. So we have $\int_{M} \operatorname{det}_{d \mu^{\prime}} \operatorname{Ric} d \mu^{\prime}=\frac{1}{a} \int_{M} \operatorname{det}_{d \mu} \operatorname{Ric} d \mu$. Thus $E(\nabla)=\int_{M} \operatorname{det} \operatorname{Ric} d \mu \int_{M} d \mu$ depends only on $\nabla \in \mathcal{A}(M)$, and $E: \mathcal{A}(M) \rightarrow \mathbf{R}$ is differentiable by virture of Corollary 2.3 .

We define a contravariant symetric 2 -tensor $\hat{R i c}=\left(\hat{R}^{i j}\right)$ as the cofactor tensor of Ric $=\left(R_{i j}\right)$. Namely

$$
\hat{R}^{i k} R_{k j}=(\operatorname{det} \operatorname{Ric}) \delta_{j}^{i} .
$$

We remark that this tensor field depends on the choice of a volume element $d \mu$. We also define $\rho$ and $\rho^{\circ}$ as

$$
\rho=\operatorname{det} \operatorname{Ric}
$$

and

$$
\rho^{\circ}=\operatorname{det} \operatorname{Ric}-\frac{\int_{M} \operatorname{det} \operatorname{Ric} d \mu}{\int_{M} d \mu}
$$


With these notations we can state the first variational formula of the functional $E$.

Proposition 3.1. $\nabla \in \mathcal{A}(M)$ is a critical point of $E: \mathcal{A}(M) \rightarrow \mathbf{R}$ if and only if the following two conditions are satisfied:

$$
\hat{R}_{; i j}^{i j}=-\frac{n+1}{n-1} \rho^{\circ}
$$

and

$$
\hat{R}_{; k}^{i j}=\frac{1}{n+1}\left(\hat{R}_{; l}^{i l} \delta_{k}^{j}+\hat{R}_{; l}^{l j} \delta_{k}^{i}\right) \text {, }
$$

where $n=\operatorname{dim} M$.

Proof. Fix $\nabla \in \mathcal{A}(M)$ with $\nabla d \mu=0$. We first consider a projective variation of $\nabla$. Let ${ }^{t} \nabla$ be defined as

$$
{ }^{t} \nabla_{X} Y=\nabla_{X} Y+t(X u) Y+t(Y u) X,
$$

where $u \in C^{\infty}(M)$ is an arbitrary function. Put

$$
d \mu(t)=e^{(n+1) t u} d \mu
$$

and we have ${ }^{t} \nabla d \mu(t)=0$. The Ricci curvature is calculated as

$$
R_{i j}(t)=R_{i j}-(n-1)\left(t u_{; i j}-t^{2} u_{; i} u_{; j}\right) .
$$

Since

we have

$$
\operatorname{det}_{d \mu(t)} \operatorname{Ric}(t) d \mu(t)=e^{-(n+1) t u} \operatorname{det}_{d \mu} \operatorname{Ric}(t) d \mu
$$

$$
\begin{aligned}
\frac{d}{d t}{ }_{\mid t=0} \operatorname{det}_{d \mu(t)} \operatorname{Ric}(t) d \mu(t) & =-(n+1) u \rho d \mu+\hat{R}^{i j} \frac{d}{d t}{ }_{\mid t=0} R_{i j}(t) d \mu \\
& =-(n+1) u \rho d \mu-(n-1) \hat{R}^{i j} u_{; i j} d \mu
\end{aligned}
$$

Hence,

$$
\frac{d}{d t}_{\mid t=0} \int_{M} \operatorname{det}_{d \mu(t)} \operatorname{Ric}(t) d \mu(t)=\int_{M} u\left(-(n+1) \rho-(n-1) \hat{R}_{; i j}^{i j}\right) d \mu .
$$

On the other hand,

$$
\frac{d}{d t} \int_{\mid t=0} d \mu(t)=(n+1) \int_{M} u d \mu \text {. }
$$

Therefore we get

$$
\left.\frac{d}{d t}_{\mid t=0} E\left({ }^{t} \nabla\right)=\int_{M} u\left(-(n+1) \rho^{\circ}\right)-(n-1) \hat{R}_{; i j}^{i j}\right) d \mu \int_{M} d \mu .
$$

The equation (3.2) follows from this formula.

From Corollary 2.2, we have only to check the variation of $\nabla$ in the direction of $S=\left(S_{j k}^{i}\right)$ with $S_{j k}^{i}=S_{k j}^{i}$ and $S_{j i}^{i}=0$. Now we put

$$
{ }^{t} \nabla_{X} Y=\nabla_{X} Y+t S(X, Y)
$$

We have ${ }^{t} \nabla d \mu=0$ for any $t$. The Ricci curvature is then

$$
R_{i j}(t)=R_{i j}+t S_{i j ; k}^{k}-t^{2} S_{i l}^{k} S_{j k}^{l} .
$$

Hence we have

$$
\begin{aligned}
{\frac{d}{d t}{ }_{\mid t=0} E\left({ }^{t} \nabla\right)} & =\int_{M} \frac{d}{d t}{ }_{\mid t=0}(\operatorname{det} \operatorname{Ric}(t)) d \mu \int_{M} d \mu \\
& =\int_{M} \hat{R}^{i j} S_{i j ; k}^{k} d \mu \int_{M} d \mu \\
& =-\int_{M} \hat{R}_{; k}^{i j} S_{i j}^{k} d \mu \int_{M} d \mu \\
& =-\int_{M}\left(\hat{R}_{; k}^{i j}-\frac{1}{n+1}\left(\hat{R}_{; l}^{i l} \delta_{k}^{j}+\hat{R}_{; l}^{l j} \delta_{k}^{i}\right)\right) S_{i j}^{k} d \mu \int_{M} d \mu
\end{aligned}
$$


The equation (3.3) follows from this formula. [

The equations (3.2) and (3.3) are not independent. In fact we can show the following.

Proposition 3.2. On a connected manifold, the equation (3.3) implies the equation (3.2).

Proof. The derivative of the determinant $\rho$ is given as $\rho_{; k}=\hat{R}^{i j} R_{i j ; k}$. This together with (3.1) yields

$$
(n-1) \rho_{; k}=\hat{R}_{; k}^{i j} R_{i j}
$$

Applying this to (3.3) we have

$$
\frac{n^{2}-1}{2} \rho_{; k}=\hat{R}_{; i}^{i j} R_{j k} .
$$

Then we get

$$
\hat{R}_{; i j k}^{i j}-\hat{R}_{; i k j}^{i j}=\hat{R}_{; i}^{i l} R_{l k j}^{j}=-\hat{R}_{; i}^{i l} R_{l k}=-\frac{n^{2}-1}{2} \rho_{; k} .
$$

On the other hand we have

$$
\begin{aligned}
\hat{R}_{; i k j}^{i j}-\hat{R}_{; k i j}^{i j} & =\left(\hat{R}^{l j} R_{l k i}^{i}+\hat{R}^{i l} R_{l k i}^{j}\right)_{; j} \\
& =\left(-\hat{R}^{l j} R_{l k}+\hat{R}^{i l} R_{l k i}^{j}\right)_{; j} \\
& =-\rho_{; k}+\hat{R}_{; j}^{i l} R^{j}{ }_{l k i}+\hat{R}^{i l} R^{j}{ }_{l k i ; j} \\
& =-\rho_{; k}+\frac{1}{n+1}\left(\hat{R}_{; m}^{i m} \delta_{j}^{l}+\hat{R}_{; m}^{m l} \delta_{j}^{i}\right) R_{l k i}^{j}-\hat{R}^{i l}\left(R^{j}{ }_{l i j ; k}+R_{l j k ; i}^{j}\right) \\
& =-\rho_{; k}-\frac{1}{n+1} \hat{R}_{; m}^{m l} R_{l k}+\hat{R}^{i l} R_{l i ; k}-\hat{R}^{i l} R_{l k ; i} \\
& =-\rho_{; k}-\frac{n-1}{2} \rho_{; k}+\rho_{; k}-\left(\hat{R}^{i l} R_{l k}\right)_{; i}+\hat{R}_{; i}^{i l} R_{l k} \\
& =\frac{(n+1)(n-2)}{2} \rho_{; k},
\end{aligned}
$$

where in the fourth equality we used the equation (3.3) and the second Bianchi identity. Hence from (3.6) and (3.7) we have

$$
\hat{R}_{; i j k}^{i j}-\hat{R}_{; k i j}^{i j}=-\frac{n+1}{2} \rho_{; k} .
$$

From (3.3) and (3.6) we have

$$
\hat{R}_{; k i j}^{i j}=\frac{1}{n+1}\left(\hat{R}_{; l i k}^{i l}+\hat{R}_{; l k j}^{l j}\right)=\frac{2}{n+1} \hat{R}_{; i j k}^{i j}+\frac{n-1}{2} \rho_{; k} .
$$

Then it is easy to see from (3.8) and (3.9) that

$$
\hat{R}_{; i j k}^{i j}=-\frac{n+1}{n-1} \rho_{; k},
$$

which implies the condition (3.2).

\section{Proof of Theorem}

It follows from (3.5) and (3.1) that

$$
\rho_{; i} \hat{R}^{i j}=\frac{2}{n^{2}-1} \rho \hat{R}_{; i}^{i j}
$$


Hence using (3.2), we have

$$
\rho_{; i j} \hat{R}^{i j}+\frac{n^{2}-3}{n^{2}-1} \rho_{; i} \hat{R}_{; j}^{i j}=-\frac{2}{(n-1)^{2}} \rho \rho^{\circ} .
$$

The proof is divided into two cases. One is the case when $n=\operatorname{dim} M$ is even. Then since Ric $\leq 0$, we

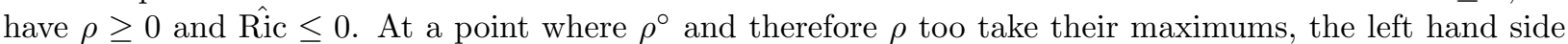
of (4.2) is nonnegative because Ric $\leq 0$, and the right hand side of (4.2) is nonpositive. Hence we conclude that $\rho^{\circ}=0$, that is, $\rho$ is a constant, which must be positive.

The other is the case when $n$ is odd. Then since Ric $\leq 0$, we have $\rho \leq 0$ and $\hat{\operatorname{Ric}} \geq 0$. At a point where $\rho$ takes its minimum, the left hand side of (4.2) is nonnegative, because $\hat{R i c} \geq 0$, and the right hand side of (4.2) is nonpostive. Hence we have $\rho^{\circ}=0$, that is, $\rho$ is constant.

In both cases we have that $\rho$ is a nonzero constant. Then it follows from $(4.1)$ that $\hat{R}^{i j}{ }_{j}=0$. Hence from (3.3), we have $\nabla$ Ric $=0$. This implies $\nabla$ Ric $=0$ because $\rho \neq 0$. Now put $g=-$ Ric, which is a Riemannian metric, and is parallel with respect to $\nabla$. Therefore $\nabla$ is the Riemannian connection of $g$.

\section{References}

[1] Besse, A. L., Einstein manifolds, Springer, 1987

[2] Kobayashi, S. and Nomizu, K., Foundation of differential geometry, Vol. 1, Interscience publishers, 1963

Department of Mathematics

Kumamoto University

Kumamoto 860-8555, Japan

e-mail: ok@math.sci.kumamoto-u.ac.jp 